\title{
HIGH RESOLUTION INTERFEROMETRIC DIGITAL ELEVATION MODEL GENERATION AND VALIDATION
}

\author{
Kapil Malik*, Dheeraj Kumar \\ Department of mining Engineering, Indian Institute of technology (ISM), Dhanbad, India.
}

Commission V, SS: Infrastructure and Development Planning

KEYWORDS: Interferometry, Cosmo-Skymed, DEM, GPS.

\begin{abstract}
:
In this paper high-resolution DEM generation has been attempted using the Cosmo-skymed data over the New Delhi, India area. The study area is an urban area filled with lots of building and settlement which was helpful to get the stable point in the time series images data. This DEM generation is based on the highly stable permanents scatter candidate (PSC). This PSC was selected on the basis of amplitude stability index which was generated/calculated using the 25 images of the cosmo-skymed acquired over the time. A sufficient number of field GPS data has been used for result validation. Interpolated output DEM at 10-meter resolution has also been compared with the available SRTM and ASTER DEM for further quality estimation. Presented result demonstrate the capabilities of the technique in constructing a high-resolution quality DEM.
\end{abstract}

\section{Introduction:}

DEM is widely used for lots of different application related to geology, geography and hydrological studies. Time to time different height model has been generated such as GTOPO, Shuttle Radar Topography Mission (SRTM), Advanced Spaceborne Thermal Emission and Reflection Radiometer (ASTER GDEM) and Carto DEM etc. GTOPO and SRTM was the USGS mission which provides the global DEM at 30-meter spatial resolution. The Ministry of Economy, Trade, and Industry (METI) of Japan and the United States National Aeronautics and Space Administration (NASA) jointly announced the release of ASTER GDEM. Carto DEM is product generated by the Indian Space Research Organization (ISRO) using the Cartosat-1 stereo data.

Since DEM has lots of importance in the different field it becomes crucial to use the best quality product. In this study, an attempt has been made to derive the best product using the state of the art interferometric technique.

\section{Data used and methodology:}

Cosmo-sky med Stripmap mode 25 images acquired between 08/06/2011 and 19/03/2015 on a regular interval has been used for this study. The constellation of small Satellites for the Mediterranean basin Observation (COSMO-SkyMed) is an Earth observation satellite system operated by the Italian Ministry of Research and Ministry of Defense and Italian Space Agency (ASI). This satellite has an X-band SAR sensor onboard. Data of this satellite is used for both military and civilian application. Spotlight, a high-resolution mode collected over a small area by steering the radar beam slightly fore-to-aft during the collection period. This satellite provides the data in spotlight, Stripmap and ScanSAR mode. Spotlight mode is a highresolution mode collected over a small area mainly for defense application. Stripmap mode is a medium-resolution mode collected over long, continuous swaths in which the beam is pointed broadside to the satellite track. ScanSAR mode is a lowresolution mode that creates extra-wide swaths by collecting short segments at different ranges and then mosaicking them together. Here is the graph showing the temporal and spatial baseline distribution of 25 images used.

Persistent scatterers (PSs) In-SAR based height estimation technique is being used here. Differential InSAR technique capable of measuring possible displacements of radar targets along the line of sight (LOS) by computing the difference of the phase values of two synthetic aperture radar (SAR) images acquired at two different times over the same area [1-5]. It's proven that interferometric data can be used to recover highresolution topographic profiles (acquiring data pairs from slightly different looking angles) [6] microwave SAR systems operating frequency is within the $1-10-\mathrm{GHz}$ band due to this even sub-centimeter range variations generate phase shifts that can be detected by the sensor. This is reason SAR is a powerful tool for precise geodetic surveys and mapping over large areas where conventional survey would be time taking.

The PS technique based on a long series of SAR data which is acquired in slightly different geometries which enable it to locate a target in 3-D space. The positioning accuracy achievable by means of the PS technique has been assessed in the order of $1 \mathrm{~m}$ in the three dimensions [8], [10].

\footnotetext{
${ }^{*}$ Corresponding author-kapil@me.ism.ac.in
} 


\section{Result and discussion:}

In This study Delhi, the Urban area is has been selected. This area is full of the urban target which ensures the presence of the PSc.

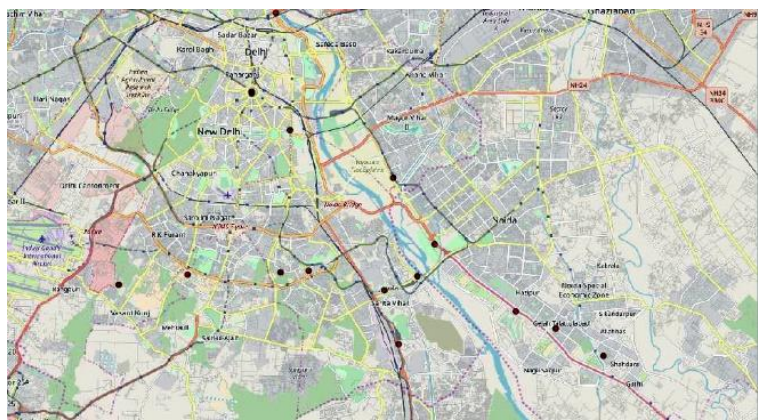

Figure-1 showing the study area and DGPS point as dotted. [Google Map]

During the process, all the DEM has been brought into the one projection system and geoid EGM08 [11] subtraction applied on the SRTM [12] and ASTER. The CARTODEM [13] and ALOS PALSAR DEM [14] are already without the geoid. In the given table-1 there is a comparison of the height value has been given

\begin{tabular}{|l|r|r|r|r|r|r|}
\hline \multicolumn{3}{|c|}{ Point ASTER ALOS_DEM } & CartoDEM & DGPS & InSAR_DEM & SRTM \\
\hline A & 166 & 162 & 162 & 160.8 & 162 & 162 \\
\hline B & 157 & 168 & 163 & 164.7 & 165 & 167 \\
\hline C & 154 & 161 & 164 & 163.6 & 164 & 163 \\
\hline D & 165.6 & 157 & 162 & 159.7 & 158 & 157 \\
\hline E & 201 & 207 & 208 & 211.2 & 209 & 207 \\
\hline
\end{tabular}

Table-1, Showing height comparison on five different control point in different height model.

\section{Conclusion:}

This is has been established in this experiment that PS-InSAR based height estimation is relatively accurate in urban DEM. More number of image provide more reliable persistent scatterer which helps us to estimate the height correctly. So this technique can be used widely after validating with more number of the control point in different terrain condition other than urban area.

\section{Reference:}

[1] R. F. Hanssen, "Radar interferometry," in Data Interpretation and Error Analysis. Dordrecht, The Netherlands: Kluwer, 2001.

[2] A. K. Gabriel, R. M. Goldstein, and H. A. Zebker, "Mapping small elevation changes over large areas: Differential radar interferometry," J. Geophys. Res., vol. 94, no. B7, pp. 91839191, 1989.j
[3] D. Massonnet and K. L. Feigl, "Radar interferometry and its application to changes in the Earth's surface," Rev. Geophys., vol. 36, no. 4, pp. 441-500, Nov. 1998.

[4] P. A. Rosen, S. Hensley, I. R. Joughin, F. K. Li et al., "Synthetic aperture radar interferometry," Proc. IEEE, vol. 88, no. 3, pp. 333-382, Mar. 2000.

[5] R. Burgmann, P. A. Rosen, and E. J. Fielding, "Synthetic aperture radar interferometry to measure Earth's surface topography and its deformation," Annu. Rev. Earth Planet. Sci., vol. 28, pp. 169-209, May 2000.

[6] S. Madsen, H. A. Zebker, and J. Martin, "Topographic mapping using radar interferometry: Processing techniques," IEEE Trans. Geosci. Remote Sens., vol. 31, no. 1, pp. 246-256, Jan. 1993.

[8] A. Ferretti, C. Prati, and F. Rocca, "Permanent scatterers in SAR interferometry," IEEE Trans. Geosci. Remote Sens., vol. 39, no. 1, pp. 8-20, Jan. 2001.

[9] Ferretti A, Prati C, Rocca F., "Non-linear subsidence rate estimation using permanent scatterers in differential SAR interferometry," IEEE Trans. Geosci. Remote Sens., vol. 38, no. 5, pp. 2202-2212, Sep. 2000.

[10] C. Colesanti, A. Ferretti, F. Novali, C. Prati, and F. Rocca, "SAR monitoring of progressive and seasonal ground deformation using the permanent scatterers technique," IEEE Trans. Geosci. Remote Sens., vol. 41, no. 7, pp. 1685-1701, Jul. 2003.

11 Pavlis, N.K., Holmes, S.A., Kenyon, S.C., and Factor, J.K., 2008. An Earth Gravitational Model to degree 2160: EGM08. Presented at the 2008 General Assembly of the European Geosciences Union, Vienna, Austria, April 12 - 18, 2008.

[12] USGS (2004) Shuttle Radar Topography Mission, 1 Arc Second scene SRTM_u03_n008e004, Unfilled Unfinished 2.0, Global Land Cover Facility, University of Maryland, College Park, Maryland, February 2000. The facility, University of Maryland, College Park, Maryland, February 2000.

[13] CartoDEM, National Remote Sensing Centre, ISRO, Government of India, Hyderabad, India.

[14] JAXA/METI 2018. ALOS PALSAR DEM 12.5m 2018 Accessed through ASF DAAC 